\title{
Análisis psicométrico de la escala de involucramiento en el trabajo de Lodahl y Kejner en trabajadores de Arequipa
}

Walter L. Arias Gallegos ${ }^{1}$ NoElia A. JimÉNEZ BARRIOS ${ }^{2}$

Resumen:

En el siguiente artículo se presenta el análisis psicométrico de la Escala de Involucramiento en el trabajo de Lodahl y Kejner, que consta de 20 ítems dispuestos en una escala tipo Likert con cinco alternativas de respuesta. Se compone de siete factores y posee adecuados niveles de validez y confiabilidad según la teoría clásica de los tests. Se aplicó la prueba a 223 trabajadores de la ciudad de Arequipa, del sector salud y del sector administrativo. Los resultados indican que la prueba tiene adecuada homogeneidad y una estructura interna de cinco factores: absorción por el trabajo, irrelevancia del trabajo, preocupación por el trabajo, sentido de trabajo y desmotivación. Sus niveles de confiabilidad se ubican por encima de 0.725 , obtenidos a través de la prueba Alfa de Cronbach. Se concluye que la prueba es válida y confiable.

Palabras clave: Involucramiento en el trabajo; validez; confiabilidad.

PSYCHOMETRICAL ANALYSIS OF LODAHL AND KeJNER'S JOB INVOLVEMENT SCALE IN WORKERS FROM AREOUIPA

Abstract:

In the following article we present a psychometric analysis of Lodahl and Kejner's Job Involvement Scale, which contains 20 items in a Likert scale with five alternatives to answer. This instrument has seven factors and adequate levels of validity and reliability according to the classic theory of tests. We applied the test to 223 workers at Arequipa City, from the health and administrative occupational field. The results indicate that the test have adequate homogeneity and an internal structure of five factors: absorption for work, irrelevance of work, concern for work, sense of work and unmotivation. Its levels of reliability are located over 0.725 , obtained by the Cronbach's Alpha Test. We conclude the test is valid and reliable.

Key words: Job involvement; validity; reliability.

\section{INTRODUCCIÓN}

El concepto de involucramiento en el trabajo fue introducido por Lodahl y Kejner en 1968, y lo definieron como el grado en el cual un individuo está absorbido psicológicamente por su trabajo (Lodahl y Kejner, 1965). Para Lawler y Hall (1970) el involucramiento en el trabajo hace referencia al grado en que un trabajador se siente identificado con su trabajo. Este constructo tiene componentes emocionales y conductuales que repercuten en el entorno laboral (Nazem y Mozaiini, 2014), así como en el trabajador (Pathak, 1983).

Más recientemente, se ha planteado que la energía, el involucramiento en el trabajo y la absorción, constituyen componentes del engagement; que se oponen a las dimensiones de agotamiento emocional, despersonalización y baja realización personal, del síndrome de burnout (Castellano, Cifré, Spontón, Medrano y Maffei, 2013). Así, mientras la despersonalización hace alusión al distanciamiento del trabajador y su cambio negativo de actitud frente al trabajo (Arias, 2015), el involucramiento implica un mayor sentido de identidad como trabajador.

Entre los principales determinantes del involucramiento en el trabajo, se tiene a las características psicológicas del trabajador y las condiciones del entorno laboral (Ekmekçi, 2011). En el primer caso, las dimensiones de extroversión, cordialidad, apertura a la experiencia, y responsabilidad de la personalidad según el modelo teórico de los Cinco Grandes, son importantes predictores del involucramiento en el trabajo (Chi-Shun y Cheng-Wen, 2009); mientras que el neuroticismo como rasgo de personalidad, tienen un impacto negativo (Mahajan, 2015). La autoeficacia también se ha relacionado positiva y significativamente con el involucramiento en el trabajo (Hui-Ling, Yu-Hsiu y Yi-Ching, 2006). De hecho, las personas que tienen elevados niveles de involucramiento en el trabajo, consideran sus obligaciones laborales como aspectos esenciales de sus vidas (Nwibere, 2014).

En el segundo caso, un clima de participación y liderazgo, donde hay autonomía y responsabilidades claras en el trabajo, con acceso a los recursos necesarios para cumplir las funciones designadas, promueven el involucramiento en el trabajo (Gilkar y Darzi, 2012). El clima organizacional también es un predictor significativo del involucramiento en el trabajo (Biswas, 2009), así como el tamaño de la empresa, de modo que mientras menos

Profesor investigador de la Universidad Católica San Pablo, Arequipa, Perú. e mail: warias@ucsp.edu.pe

2 Licenciada en administración de empresas por la Universidad Católica de Santa María, Arequipa, Perú. e mail: noelia.jimenez@ucsm.edu.pe 
grande es la empresa, mayor es el involucramiento (Deosthalee, 2004). La naturaleza de la labor desempeñada, puede ser otro factor importante que promueve el involucramiento, como suele ser el caso de las profesoras de nivel preescolar, que se sienten emocionalmente reforzadas por el contacto que tienen con niños pequeños y esto incrementa su involucramiento (Ching-Sheue, 2015). En ese sentido, la implicación emocional del trabajador, conlleva al involucramiento en el trabajo (Lucia-Casademunt, Morales-Gutiérrez y Ariza-Montes, 2012)

Así, el involucramiento en el trabajo es una de las más importantes características que todo trabajador debe poseer, debido a que se asocia con una mayor productividad. De hecho, los trabajadores que no poseen involucramiento laboral se tornan apáticos y carentes de iniciativa (Huselid, \& Day, 1991). En ese sentido, diversos estudios han reportado relaciones positivas entre el involucramiento en el trabajo y la satisfacción laboral (Saeidi e Iravani, 2016), la motivación intrínseca (Lowler y Hall, 1970), el compromiso en el trabajo (Blau y Boal, 1987), el apoyo social (Mishra y Shyam, 2005), el liderazgo transformacional (Nazem y Mozaiini, 2014), la cultura organizacional (Rizvi, 2013), el civismo organizacional (Saxena y Saxena, 2015); y relaciones negativas con el estrés marital (Uzonwanne, 2014), la inestabilidad emocional (Mahajan, 2015) y el estrés laboral (López-Araujo, Osca y Peiró, 2007).

Conceptualmente, el involucramiento en el trabajo se suele confundir con el compromiso en el trabajo, sin embargo, son dos constructos diferentes, pero íntimamente relacionados (Khan, Jam, Akbar, Khan y Hijazi, 2011). Mientras el compromiso organizacional es el apego afectivo por los valores y las metas de la organización, el involucramiento laboral hace referencia al grado en que un individuo se orienta o está determinado por su trabajo. Además, mientras el compromiso laboral puede valorarse a través de la lealtad hacia la organización, el involucramiento en el trabajo se evidencia más en la productividad. Es decir, que, mientras en el compromiso laboral, se valora cómo el individuo se vincula con la organización, el involucramiento laboral tiene que ver con cómo el trabajo tiene prioridad en la vida de la persona (Ekmekçi, 2011). Asimismo, el compromiso laboral suele incrementarse con el tiempo, sobre todo en las mujeres (Sancerni, Peiró, González-Roma y Meliá, 1989); mientras que el involucramiento en el trabajo se mantiene estable (Chi-Shun y Cheng-Wen, 2009).

En el Perú no son comunes los estudios sobre el compromiso laboral. Por ejemplo, Alejandro Loli Pineda ha desarrollado varios estudios sobre este constructo en la ciudad de Lima. En un primer estudio, se reportó que el compromiso afectivo se rela- ciona de manera alta y significativa con la satisfacción en el trabajo en 190 trabajadores de diferentes empresas de la capital (Arias, Varela, Loli y Quintana, 2003). En otro estudio con 205 trabajadores administrativos de 20 facultades de una universidad pública de Lima, encontró que el compromiso con el trabajo y el compromiso afectivo se relacionan con el sentimiento de pertenencia del trabajador y la jerarquía del puesto (Loli, 2006). Finalmente, en un tercer estudio, Loli (2008) encontró que el nivel de compromiso organizacional no se relacionaba con las variables demográficas de los trabajadores.

Con respecto al involucramiento en el trabajo, no existen estudios previos publicados en el país, quizás porque no se cuenta con instrumentos validados en nuestro medio. En ese sentido, son pocos los instrumentos diseñados para valorar el involucramiento en el trabajo. Uno de ellos es el Job Involvement Scale de Lodahl y Kejner (1965), que consta de 20 ítems con cinco alternativas de respuesta en una escala tipo Likert, con una confiabilidad con el método de consistencia interna de 0.82 . otro instrumento es el Job Involvement Questionnaire de Kanungo (1982), pero el inventario de Lodahl y Kejner ha sido es más utilizado en países de habla hispana (Betanzos y Paz, 2007).

En el caso de Latinoamérica, el primer estudio que se basa en el Job Involvement Scale de Lodahl y Kejner, fue el que realizó Martínez-Lugo (1988) en Puerto Rico, con una muestra de 87 trabajadoras de una fábrica de ensamble de interruptores, reportando que, a mayor edad, las mujeres de su muestra tenían mayor involucramiento en el trabajo. En otro estudio con 174 participantes, el mismo autor, encontró que el involucramiento en el trabajo se relacionaba de manera positiva con la cohesión, el apoyo del superior, la autonomía, la claridad de las funciones, la innovación, la comodidad física en el trabajo y la satisfacción laboral (Martínez-Lugo, 1990).

Pocos años después, Andújar y Martínez-Lugo (1996) presentaron el primer análisis psicométrico de la escala de Lodahl y Kejner de involucramiento en el trabajo, en el que reportaron buenas cualidades psicométricas del instrumento desde la teoría clásica de los test. En concreto, hallaron que la prueba tiene buena homogeneidad, pues las correlaciones ítem-test fueron superiores a 0.492 . Además, contaba con una estructura interna de siete factores: Factor negativo, Preocupación por el trabajo, Proactividad, Sentido del trabajo, Importancia del trabajo, Compromiso y Fluir en el trabajo; que explicaron el $65.8 \%$ de la varianza total de la prueba. Sin embargo, al valorar las cualidades psicométricas de la escala con el modelo de Samejima de la teoría psicométrica de respuesta al ítem, los autores, indicaron que dicho instrumento no se 
ajustaba adecuadamente a la muestra con la que se había trabajado.

En base a todos estos alcances, se propone valorar las propiedades psicométricas de la Escala de Involucramiento en el Trabajo de Lodahl y Kejner, mediante la teoría clásica de los test; por lo tanto, el presente estudio es de carácter instrumental (León y Montero, 2007).

\section{MÉTODO}

\subsection{Participantes}

La muestra está constituida por 223 personas de las áreas laborales de la salud $(n=148)$ y la administración $(n=75)$. El $49.3 \%$ son mujeres y el $50.7 \%$ son varones, con una edad media de 36.97 años y una desviación estándar de \pm 14 .8 dentro de un rango de 18 y 69 años. La muestra fue seleccionada a través de la técnica de grupos intactos, por tanto, se empleó un muestreo no probabilístico (Hernández, Fernández y Baptista, 2011).

\subsection{Instrumentos}

Se ha utilizado la Escala de Involucramiento en el Trabajo de Lodahl y Kejner que consta de 20 reactivos con cinco alternativas de respuesta en una escala tipo Likert que va desde muy de acuerdo (5) hasta muy desacuerdo (1). De los 20 ítems, 8 se puntúan de manera inversa $(9,10,13,16,17$, 18, 19 y 20). Tiene una estructura de siete factores (Andújar y Martínez-Lugo, 1996): Factor negativo (ítems 10, 13, 18 y 19), Preocupación por el trabajo (ítems 7, 9 y 2), Proactividad (ítems 5, 6 y 12), Sentido del trabajo (ítems 1, 2 y 3), Importancia del trabajo (ítems 11 y 14), Compromiso (ítems 16 y 17) y Fluir en el trabajo (ítems 4,8 y 15). Este instrumento cuenta con validez y confiabilidad, reportados por Andújar y Martínez-Lugo (1996) para muestras latinoamericanas.

\subsection{Procedimientos}

La aplicación del instrumento se hizo entre trabajadores de organizaciones públicas dedicadas asistencia de salud y la atención de usuarios. Las evaluaciones se realizaron previa autorización de las autoridades competentes en cada caso, y en el horario de trabajo de los participantes, según las coordinaciones correspondientes. Los datos se procesaron siguiendo los lineamientos de la teoría clásica de los tests y a través de la aplicación del programa estadístico SPSS versión 21.

\section{RESULTADOS}

Con la finalidad de valorar la homogeneidad de los ítems que componen la Escala de Involucramiento en el Trabajo de Lodahl y Kejner, se realizaron correlaciones ítem-test entre los puntajes obtenidos en cada ítem y la puntuación total de la prueba. Todos los ítems obtuvieron correlaciones superiores a 0.2 , siendo el rango de los coeficientes de correlación desde 0.297 para el ítem 10 "Tengo otras actividades más importantes que mi trabajo" y de 0.577 para el ítem 6 "Las cosas más importantes que me suceden guardan relación con mi trabajo".

En la Tabla 1 se pueden apreciar las medias y la desviación estándar de cada ítem, así como los índices de correlación ítem-test. Ningún ítem fue eliminado, pues se corrobora que los ítems son representativos del constructo involucramiento en el trabajo.

Tabla 1. Correlaciones ítem-test

\begin{tabular}{|c|c|c|c|}
\hline $\mathbf{N}^{\mathbf{S}}$ & Media & $\mathbf{D E}$ & $\mathbf{r}$ \\
\hline $\mathbf{1}$ & 3.475 & 1.258 & 0.544 \\
\hline $\mathbf{2}$ & 4.336 & 0.981 & 0.529 \\
\hline $\mathbf{3}$ & 3.596 & 1.188 & 0.557 \\
\hline $\mathbf{4}$ & 4.026 & 0.976 & 0.517 \\
\hline $\mathbf{5}$ & 3.901 & 0.990 & 0.564 \\
\hline $\mathbf{6}$ & 3.390 & 1.084 & 0.577 \\
\hline $\mathbf{7}$ & 2.919 & 1.231 & 0.524 \\
\hline $\mathbf{8}$ & 3.663 & 1.098 & 0.444 \\
\hline $\mathbf{9}$ & 3.322 & 1.144 & 0.365 \\
\hline $\mathbf{1 0}$ & 3.282 & 1.109 & 0.297 \\
\hline $\mathbf{1 1}$ & 2.766 & 1.237 & 0.477 \\
\hline $\mathbf{1 2}$ & 3.313 & 1.170 & 0.485 \\
\hline $\mathbf{1 3}$ & 3.174 & 1.241 & 0.385 \\
\hline $\mathbf{1 4}$ & 3.493 & 1.138 & 0.411 \\
\hline $\mathbf{1 5}$ & 3.955 & 0.980 & 0.459 \\
\hline $\mathbf{1 6}$ & 3.255 & 1.107 & 0.359 \\
\hline $\mathbf{1 7}$ & 3.134 & 1.102 & 0.377 \\
\hline $\mathbf{1 8}$ & 3.139 & 1.045 & 0.468 \\
\hline $\mathbf{1 9}$ & 3.394 & 1.045 & 0.401 \\
\hline $\mathbf{2 0}$ & 3.067 & 1.332 & 0.451 \\
\hline
\end{tabular}

Fuente: Elaboración propia.

Seguidamente, se procedió a realizar un análisis factorial exploratorio, con la finalidad de valorar la validez de constructo de la Escala de Involucramiento en el Trabajo de Lodahl y Kejner. En la Tabla 
2 se observan las medidas de adecuación del análisis factorial, mediante la prueba Kaiser-Meyer-Olkim, obteniéndose un valor de 0.769 y medidas de la prueba de esfericidad de Bartlet que resultaron significativas $(x 2=1149.702 ; g l=231 ; p<0.000)$, lo que sugiere que la escala tiene una estructura conformada por varias dimensiones.

Tabla 2. Medidas de adecuación de las variables al análisis factorial

\begin{tabular}{|c|r|l|}
\hline $\begin{array}{c}\text { Medida de adecuación muestral } \\
\text { de Kaiser-Meyer-Olkin }\end{array}$ & KMO & 0.769 \\
\hline Prueba de esfericidad & $\begin{array}{r}\text { Chi-cuadrado } \\
\text { aproximado }\end{array}$ & 1149.702 \\
de Bartlet & gl & 190 \\
Sig. & 0.000 \\
\hline
\end{tabular}

Fuente: Elaboración propia.

En la Tabla 3 se tienen los porcentajes de la varianza explicada, a través de la suma de las extracciones elevadas al cuadrado y la suma de las saturaciones elevadas al cuadrado tras la rotación varimax de los autovalores superiores a 1, que indican que la prueba se compone cinco factores distinguibles, que explican el $54.75 \%$ de la varianza total de la prueba. El primer factor explica el $15.94 \%$ de la varianza total, el segundo factor explica el $11.29 \%$, el tercer factor el $10.58 \%$, el cuarto factor el $8.69 \%$ y el quinto factor explica el $8.24 \%$ de la varianza total de la prueba.

El análisis factorial por medio del método de componentes principales reporta que la estructura factorial de la Escala de Involucramiento en el Trabajo de Lodahl y Kejner se compone de cinco factores que hemos denominado de la siguiente manera:

F1: Absorción por el trabajo (ítems 1, 2, 4, 5, 8 y 15)

F2: Irrelevancia del trabajo (ítems 10, 14, 18 y 19)

F3: Preocupación por el trabajo (ítems 7, 11 y 12)

F4: Sentido de trabajo (ítems 3, 6, 9 y 20)

F5: Desmotivación (ítems 13, 16 y 17)

Básicamente, los factores originales se reconstituyeron, dando lugar a que el factor negativo y algunos ítems inversos constituyan el factor Irrelevancia del trabajo y Desmotivación; mientras que el factor Absorción por el trabajo comprende ítems de los factores originales (según Andújar y Martínez-Lugo, 1996) de Fluir en el trabajo, Sentido del trabajo y Proactividad; el de Preocupación por el trabajo comprende ítems de los factores originales de Preocupación, Proactividad y Sentido de trabajo; y el factor de Sentido del trabajo abarca ítems de los factores Proactividad, Sentido y Preocupación por

Tabla 3. Porcentajes de varianza explicada

\begin{tabular}{|c|c|c|c|c|c|c|c|c|c|}
\hline \multirow{2}{*}{ Factores } & \multicolumn{3}{|c|}{ Autovalores iniciales } & \multicolumn{3}{|c|}{$\begin{array}{l}\text { Sumas de las saturaciones al cuadrado } \\
\text { de la extracción }\end{array}$} & \multicolumn{3}{|c|}{$\begin{array}{l}\text { Suma de las saturaciones al cuadrado } \\
\text { de la rotación }\end{array}$} \\
\hline & Total & $\begin{array}{c}\% \text { de la } \\
\text { varianza }\end{array}$ & $\%$ acumulado & Total & $\begin{array}{l}\% \text { de la va- } \\
\text { rianza }\end{array}$ & $\%$ acumulado & Total & $\begin{array}{l}\% \text { de la va- } \\
\text { rianza }\end{array}$ & $\%$ acumulado \\
\hline 1 & 4.468 & 22.342 & 22.342 & 4.468 & 22.342 & 22.342 & 3.190 & 15.949 & 15.949 \\
\hline 2 & 2.398 & 11.988 & 34.330 & 2.398 & 11.988 & 34.330 & 2.260 & 11.298 & 27.247 \\
\hline 3 & 1.711 & 8.555 & 42.885 & 1.711 & 8.555 & 42.885 & 2.116 & 10.580 & 37.827 \\
\hline 4 & 1.250 & 6.251 & 49.136 & 1.250 & 6.251 & 49.136 & 1.738 & 8.690 & 46.517 \\
\hline 5 & 1.124 & 5.622 & 54.758 & 1.124 & 5.622 & 54.758 & 1.648 & 8.241 & 54.758 \\
\hline 6 & .996 & 4.982 & 59.740 & & & & & & \\
\hline 7 & .909 & 4.547 & 64.288 & & & & & & \\
\hline 8 & .892 & 4.458 & 68.746 & & & & & & \\
\hline 9 & .821 & 4.107 & 72.853 & & & & & & \\
\hline 10 & .720 & 3.601 & 76.454 & & & & & & \\
\hline 11 & .667 & 3.337 & 79.790 & & & & & & \\
\hline 12 & .613 & 3.067 & 82.857 & & & & & & \\
\hline 13 & .547 & 2.734 & 85.591 & & & & & & \\
\hline 14 & .539 & 2.697 & 88.288 & & & & & & \\
\hline 15 & .508 & 2.542 & 90.830 & & & & & & \\
\hline 16 & .450 & 2.248 & 93.078 & & & & & & \\
\hline 17 & .406 & 2.030 & 95.108 & & & & & & \\
\hline 18 & .361 & 1.804 & 96.912 & & & & & & \\
\hline 19 & .339 & 1.697 & 98.609 & & & & & & \\
\hline 20 & .278 & 1.391 & 100.000 & & & & & & \\
\hline
\end{tabular}

Fuente: Elaboración propia. 
el trabajo. Las nuevas denominaciones por tanto provienen del contenido de los ítems y su nueva estructura factorial (ver Tabla 4).

Tabla 4. Matriz de componentes con rotación varimax

\begin{tabular}{|c|c|l|l|l|l|}
\hline ítems & Factor 1 & Factor 2 & Factor 3 & Factor 4 & Factor 5 \\
\hline 01 & 0.428 & & & & \\
\hline 02 & 0.812 & & & & \\
\hline 03 & & & & 0.542 & \\
\hline 04 & 0.759 & & & & \\
\hline 05 & 0.724 & & & & \\
\hline 06 & & & & 0.531 & \\
\hline 07 & & & 0.692 & & \\
\hline 08 & 0.569 & & & & \\
\hline 09 & & & & 0.644 & \\
\hline 10 & & 0.761 & & & \\
\hline 11 & & & 0.794 & & \\
\hline 12 & & & 0.621 & & \\
\hline 13 & & & & & 0.589 \\
\hline 14 & & 0.441 & & & \\
\hline 15 & 0.482 & & & & \\
\hline 16 & & & & & 0.779 \\
\hline 17 & & & & & 0.395 \\
\hline 18 & & 0.736 & & & \\
\hline 19 & & 0.648 & & & \\
\hline 20 & & & & 0.683 & \\
\hline
\end{tabular}

Fuente: Elaboración propia

Finalmente, en la Tabla 5 se presentan las correlaciones entre los cinco factores, que son bajas entre el Factor 1 y el Factor 2, el Factor 2 y el Factor 4, el Factor 4 y el Factor 5 . Las correlaciones moderadas fueron entre el Factor 1, 3 y 4 , el Factor 2 y 5 , el Factor 3 y 4 ; y las correlaciones entre cada uno de los factores y el total de la prueba fueron superiores a 0.518 , lo que sugiere que los factores se relacionan con el constructo de involucramiento en el trabajo. En la Tabla 5 también se aprecian las medias obtenidas y sus desviaciones estándar de cada uno de los cinco factores, y los índices de confiabilidad, obtenidos por medio del método de consistencia interna, a través de la prueba Alfa de Cronbach. Los valores obtenidos con adecuados púes superan el 0.7 , siendo el Factor 3, el que obtiene índices de confiabilidad más altos $(\alpha=0.803)$.

Tabla 5. Correlaciones entre factores y coeficientes de confiabilidad

\begin{tabular}{|l|r|r|r|r|r|r|}
\hline & \multicolumn{1}{|c|}{ F1 } & \multicolumn{1}{c|}{ F2 } & F3 & F4 & F5 & Total \\
\hline F1 & 1 & & & & & \\
\hline F2 & 0.218 & 1 & & & & \\
\hline F3 & 0.438 & 0.142 & 1 & & & \\
\hline F4 & 0.414 & 0.216 & 0.425 & 1 & & \\
\hline F5 & 0.157 & 0.368 & 0.162 & 0.201 & 1 & \\
\hline Total & 0.758 & 0.573 & 0.659 & 0.709 & 0.518 & 1 \\
\hline X & 23.358 & 13.309 & 9 & 13.376 & 9.565 & 68.609 \\
\hline DS & 4.230 & 2.980 & 2.734 & 3.253 & 2.486 & 10.311 \\
\hline a & 0.767 & 0.772 & 0.803 & 0.771 & 0.785 & 0.725 \\
\hline
\end{tabular}

Fuente: Elaboración propia.
Este análisis psicométrico permite concluir que Escala de Involucramiento en el Trabajo de Lodahl y Kejner, presenta propiedades psicométricas adecuadas de validez y confiabilidad, a pesar de que la estructura factorial obtenida por Andújar y Martínez-Lugo (1996) de siete factores, se redujo a solo cinco.

\section{DISCUSIÓN}

De acuerdo con la teoría clásica de los tests, el puntaje total de una prueba, refleja la valoración de la habilidad, rasgo, actitud, destreza, etc. que se desea medir (Magnuson, 1969). Sin embargo, este supuesto reposa en el concepto de validez y confiabilidad, entendiendo por el primero, el grado en que una prueba mide lo que se supone que debe medir; mientras que la confiabilidad se refiere a la consistencia que presenta los valores de la prueba en el tiempo (Kaplan y Saccuzzo, 2006). Además, existen diversos tipos de validez que aluden a formas y métodos diferentes para obtener los criterios de precisión del instrumento (Muñiz, 2005).

Una de las formas de validez que tiene especial relevancia, es la denominada validez de constructo (Livia y Ortiz, 2014), que se obtiene a través del análisis factorial, con la finalidad de valorar la estructura interna de un instrumento (Yela, 1957). En otras palabras, la validez de constructo se refiere a cómo los ítems de un instrumento se agrupan en un factor, que sería indicador de su afinidad temática. Por otro lado, lo deseable es que un instrumento tenga una estructura factorial unidimensional (Burga, 2006), pero cuando el constructo con el que se trabaja es complejo, suele componerse de dos o más dimensiones, cuyo ajuste factorial, reflejaría la validez estructural que lo define (Fleming y Merino, 2005).

Para el caso, de la presente investigación, se ha sometido a análisis psicométrico, la escala de Involucramiento en el Trabajo de Lodahl y Kejner, con el fin de valorar su validez y confiabilidad, y de este modo pueda ser aplicada en el ámbito local, ya que el involucramiento en el trabajo, es una variable que ha capturado la atención de los hombres de empresa, en los últimos años. Nuestros resultados indican que la Escala de Involucramiento es un instrumento homogéneo, por cuanto todos los reactivos se han correlacionado de manera positiva y con coeficientes de correlación, por encima de 0.297 , con la puntuación total de la prueba, lo que supone que todos los ítems miden el mismo constructo. Estos resultados han sido similares a los reportados por Lodahl y Kejner (1965) para la versión original anglosajona, y por Andújar y Martínez-Lugo (1996) para la versión latinoamericana. Sin embargo, en el primer caso, las correlaciones ítem-test, fueron 
todas mayores a 0.3 , mientras que, en el segundo caso, las correlaciones ítem-test fueron negativas para dos ítems.

Por otro lado, si bien ambas versiones obtuvieron una estructura factorial de siete dimensiones, en nuestro estudio se redujeron a solo cinco, al igual que otras revisiones del instrumento han reportado solo cinco (Schwyhart y Smith, 1972) o tres factores (Hoole y Boshoff, 1998). De hecho, una estructura de tres factores para el constructo de involucramiento en el trabajo, suele ser más parsimoniosa (Bentazos y Paz, 2007). La cuestión de fondo, empero, es que el involucramiento en el trabajo suele confundirse, tanto teórica como psicométricamente, con el compromiso laboral y la motivación intrínseca (Morrow, 1993); aunque sus índices de confiabilidad han sido siempre adecuados (Ramsey, Lask y Marshall, 1995), como también se ha visto en el presente estudio.

Con respecto a su estructura, la solución de los cinco factores resultantes, comprende dos dimensiones negativas (irrelevancia del trabajo y desmotivación), a diferencia de la versión original y la validada en Puerto Rico, en las que solo se aprecia un factor negativo. Los otros tres factores, absorción por el trabajo, preocupación por el trabajo y sentido de trabajo; obtienen correlaciones positivas y significativas entre sí, superiores a 0.4 , lo que sugiere que se encuentran moderadamente relacionadas. En tal sentido, el involucramiento en el trabajo, de acuerdo a nuestros resultados, podría hacer alusión al grado en que una persona está absorta con sus quehaceres laborales, porque tienen sentido para él y le demandan cierto grado de preocupación. Pero esto también implica que habría que diferenciar este constructo de otros conceptos como el "trabajolismo" o adicción al trabajo, ya que cuando se realizaron los primeros estudios psicométricos de la escala de Lodahl y kejner, dicho constructo no estaba todavía difundido como hoy en día.

De las tres dimensiones halladas, la de Absorción en el trabajo, corresponde con la dimensión de Fluir de la escala original, y que comprende ítems de los factores Fluir en el trabajo, Sentido del trabajo y Proactividad; explicando el $15.94 \%$ de la varianza total de la prueba, es decir, la mayor cantidad de varianza en relación a los demás factores. De manera que, es posible que este factor sea el que presenta mayor peso explicativo del involucramiento en el trabajo, no solo psicométricamente, sino a nivel teórico, ya que de acuerdo con Csikszentmihalyi (2003), la capacidad de fluir en el trabajo, se relaciona con cualidades tales como innovación, compromiso, responsabilidad, disfrute y autorrealización.
Sin embargo, hace falta mayor investigación sobre este tema, ya que, en el país, aunque se reconoce la importancia del involucramiento laboral, no se han realizado estudios previos sobre este constructo, como sí ha ocurrido con otras variables como estrés laboral, satisfacción laboral, clima organizacional, síndrome de burnout, etc. (León, 2013). En ese sentido, la presente investigación hace una contribución teórica y metodológica, al introducir el estudio del involucramiento en el trabajo en el Perú, y al presentar un instrumento validado en la ciudad de Arequipa, que puede ser objeto de estudio y de aplicación en contextos culturales similares dentro del país.

Por otro lado, algunas consideraciones deben ser tomadas en cuenta para superar algunas limitaciones del presente trabajo. Entre estas tenemos el tamaño de la muestra, ya que, sería recomendable tomar muestras más representativas y contar con una versión estandarizada del instrumento. Asimismo, es importante aplicar análisis estadísticos más rigurosos, pues solo se ha trabajado con el análisis factorial exploratorio, pero un análisis factorial confirmatorio, podría darnos más luces sobre la estructura interna del instrumento, con indicadores de ajuste más precisos. En ese sentido, sería de gran relevancia teórica, el poder valorar las relaciones del involucramiento en el trabajo con otras variables organizacionales, con la finalidad de evaluar sus manifestaciones, su causalidad y su poder explicativo en el contexto laboral.

Finalmente, solo queda recalcar que esta es una primera aproximación a este tema, y que no se agota con el presente trabajo, sino que, por el contrario, debe ser un punto de partida para iniciar una línea de investigación en el país, sobre este fenómeno complejo y afín las nuevas tendencias del comportamiento organizacional positivo, que abarca constructos relacionados como el civismo organizacional, el fluir (o flow), el engagement y una diversidad de emociones positivas en el trabajo como la felicidad, la gratitud, la esperanza, la espiritualidad, etc.

\section{CONCLUSIONES}

En primer lugar, se puede concluir que la Escala de Involucramiento en el Trabajo de Lodahl y Kejner es homogénea en cuanto a las correlaciones ítemtest obtenidas, pues todos los coeficientes superan el mínimo 0.2, lo que supone que todos los ítems miden el mismo constructo.

En segundo lugar, la escala cuenta con una estructura factorial de cinco dimensiones que explican el $54.75 \%$ de la varianza total, pero no se han corro- 
borado los siete factores de la escala original trabajada por Andújar y Martínez-Lugo (1996).

En tercer lugar, la prueba cuenta con índices adecuados confiabilidad para el contexto laboral arequipeño, pues se obtuvieron índices de consistencia interna superiores a 0.7 mediante la prueba alfa de Cronbach.

\section{REFERENCIAS}

[1] Andújar, C. A. y Martínez-Lugo, M. E. (1996). El nivel en que se involucran los empleados en el trabajo. Análisis psicométrico de la Escala de Lodahl y Kejner mediante las teorías clásica y moderna de la medición. Revista Latinoamericana de Psicología, 28(1), 109-134.

[2] Arias, F., Varela, D., Loli, A. y Quintana, M. (2003). El compromiso organizacional y su relación con algunos factores demográficos y psicológicos. Revista de Investigación en Psicología, 6(2), 13-25.

[3] Arias, W. L. (2015). Síndrome de burnout y afrontamiento. Arequipa: Adrus.

[4] Betanzos, N. y Paz, F. (2007). Análisis psicométrico del compromiso organizacional como variable actitudinal. Anales de Psicología, 23(2), 207-215

[5] Biswas, S. (2009). Psychological climate as a predictor of job involvement. Management Insight, 5(1), 74-79.

[6] Blau, G. J. y Boal, K. B. (1987). Conceptualizing how job involvement and organizational commitment affects turnover and absenteeism. The Academy of Management Review, 12, 288-300.

[7] Burga, A. (2006). La unidimensionalidad de un instrumento de medición: perspectiva factorial. Revista de Psicología de la PUCP, 24(1), 5380.

[8] Castellano, E., Cifré, E., Spontón, C., Medrano, L. A. y Maffei, L. (2013). Emociones positivas y negativas en la predicción del burnout y engagement en el trabajo. Revista Peruana de Psicología y Trabajo Social, 2(1), 75-88.

[9] Ching-Sheue, F. U. (2015). The effect of emotional labor on job involvement in preschool teachers: Verifying the mediating effect of psychological capital. The Turkish Online Journal of Education Technology, 14(3), 145-156.

[10] Chi-Shun, L. y Cheng-Wen, L. (2009). An empirical study of employee job involvement and personality traits: The case of Taiwan. International Journal of Economics and Management, 3(1), 22-36.
[11] Csikszentmihalyi, M. (2003). Fluir en los negocios. Barcelona: Kairós.

[12] Deosthalee, P. G. (2004). Relationship of work involvement with size of company, nature of appointment, and length of service employees. Pakistan Journal of Psychological Research, 19(1-2), 15-23.

[13] Ekmekçi, A. K. (2011). A study on involvement and commitment of employees in Turkey. Journal of Public Administration and Policy Research, 3(3), 68-73.

[14] Fleming, J. S. y Merino, C. (2005). Medidas de simplicidad y de ajuste factorial: un enfoque para la evaluación de escalas construidas factorialmente. Revista de Psicología de la PUCP, 23(2), 249-266.

[15] Gilkar, N. A. y Darzi, J. A. (2012). Employee job involvement and sense of participation influence on job satisfaction: an empirical evidence. International Journal of economics and Management Resecarch, 2(8), 1-8.

[16] Hernández, R., Fernández, C. y Baptista, P. (2011). Metodología de la investigación. México: McGraw-Hill.

[17] Hoole, C. y Boshoff, A. B. (1998). The job involvement construct and its measurement. South African Journal of Economics and Management Studies, 1(2), 331-347.

[18] Hui-Ling, Y., Yu-Hsiu, K. y Yi-Ching, H. (2006). The job self-efficacy and job involvement of clinical nursing teachers. Journal of Nursing Research, 14(3), 237-248.

[19] Huselid, M. A. y Day, N. E. (1991). Organizational commitment, job involvement, and turnover: a substantial and methodological analysis. Journal of Applied Psychology, 76(3), 380-391.

[20] Kanungo, R. N. (1982). Measurement of job and work involvement. Journal of Applied Psychology, 67(3), 341-349.

[21] Kaplan, R. M., \& Saccuzzo, D. P. (2006). Pruebas psicológicas. Principios, aplicaciones y temas. México: Thomson.

[22] Khan, T. I., Jam, F. A., Akbar, A., Khan, M. B. y Hijazi, S. T. (2011). Job involvement as predictor of employee commitment: Evidence from Pakistan. International Journal of Business and Management, 6(4), 252-262.

[23] Lawler, E. y Hall, D. (1970). Relationship of job characteristics to job involvement, satisfaction, and intrinsic motivation. Journal of Applied Psychology, 54, 305-312. 
[24] León, F. (2013). Las psicologías en el área organizacional-social en el Perú (2003-2012). Revista de Psicología de la PUCP, 31(2), 117226.

[25] León, I. y Montero, O. (2007). A guide for name studies in psychology. International Journal of Clinical and Health Psychology, 7(3), 847-862.

[26] Livia, J. y Ortiz, M. (2014). Construcción de pruebas psicométricas: Aplicaciones a las ciencias sociales y de la salud. Lima: Universidad Nacional Federico Villarreal.

[27] Lodahl, T. M. y Kejner, M. (1965). The definition and measurement of job involvement. Journal Applied Psychology, 49, 24-33.

[28] Loli, A. (2006). Compromiso organizacional de los trabajadores de una universidad pública de Lima y su relación con algunas variables demográficas. Revista de Investigación en Psicología, 9(1), 37-67.

[29] Loli, A. (2008). Compromiso organizacional de los trabajadores de una universidad pública. Producción y Gestión, 7, 1-8.

[30] López-Araujo, B., Osca, A. y Peiró, J. M. (2007). El papel modulador de la implicación con el trabajo en la relación entre el estrés y la satisfacción laboral. Psicothema, 19(1), 81-87.

[31] Lucia-Casademunt, A. M.; Morales-Gutiérrez, A. C. y Ariza-Montes, J. A. (2012). La implicación emocional en el puesto de trabajo: Un estudio empírico. Intangible Capital, 8(2), 364405.

[32] Magnusson, D. (1969). Teoría de los tests. México: Trillas.

[33] Mahajan, K. (2015). An empirical study of job involvement and personality traits. International Journal of Scientific Research, 4(1), 56-58.

[34] Martínez-Lugo, M. E. (1988). La involucración con el trabajo y su relación con la edad, el locus de control y los años de servicio. Revista Latinoamericana de Psicología, 20(2), 137148.

[35] Martínez-Lugo, M. E. (1990). Variables asociadas a la involucración en el trabajo. Revista Latinoamericana de Psicología, 22(2), 211222.

[36] Mishra, P. C. y Shyam, M. (2005). Social support and job involvement in prison officers. Journal of the Indian Academy of Applied Psychology, 31(1-2), 7-11.

[37] Morrow, P. C. (1993). The theory and measurement of work commitment. Inglaterra: JAI Press.

[38] Muñiz, J. (2005). La validez desde una óptica psicométrica. Acta Comportamentalia, 13(1), 9-20.
[39] Nazem, F. y Mozaiini, M. (2014). Investigating the relationship between leadership style (transformational and pragmatic) and employee's job involvement at Islamic Azad University of Roudehen, Damavand, Pardis, Boomehen and Firuzkuh to provide an appropriate model. Bulletin of Environmental, Pharmacology and Life Sciences, 3(4), 82-88.

[40] Nwibere, B. M. (2014). Interactive relationship between job involvement, job satisfaction, organizational citizenship behavior, and organizational commitment in Nigerian universities. International Journal of Management and Sustainability, 3(6), 321-340.

[41] Pathak, R. D. (1983). Job involvement and need satisfaction of bank officers in India. Research Note, 8(4), 297-301.

[42] Ramsey, R., Lask, F. G. y Marshall, G. W. (1995). A critical evaluation of a measure of job involvement: The use of the Lodahl and Kejner scale with salespeople. Journal of Personnel Selling and Sales Management, 315(3), 65-74.

[43] Rizvi, F. M. (2013). Job involvement as related to organizational culture and social support among nurses of private and government hospitals. Interdisciplinary Journal of Contemporary Research in Business, 5(7), 564-574.

[44] Saeidi, K. e Iravani, M. R. (2016). Predicting the job involvement based on job motivation variable among experts and supervisors of Isfahan Cement Company. The Caspian Sea Journal, 10(1), 278-282.

[45] Sancerni, M. D., Peiró, J. M., González-Roma, V. y Meliá, J. L. (1989). El compromiso con el trabajo en la transición de la escuela al mundo laboral. Revista de Psicología Social, 4(3), 247-259.

[46] Saxena, S. y Saxena, R. (2015). Impact of job involvement and organizational commitment on organizational citizenship behavior. International Journal of Management, Business and Research, 5(1), 19-30.

[47] Schwyhart, W. R. y Smith, P. C. (1972). Factors in the job involvement of middle managers. Journal of Applied Psychology, 56, 227233.

[48] Uzonwanne, F. C. (2014). Marital stress and job involvement among pilots and other employees at an Air Force Base in Lagos, Nigeria. European Journal of Humanities and Social Sciences, 31(1), 1-22.

[49] Yela, M. (1957). La técnica del análisis factorial. Un método de investigación en psicología y pedagogía. Madrid: Biblioteca Nueva. 